\title{
Detection of Distinct Human Immunodeficiency Virus Type 1 Circulating Recombinant Forms in Northeast Brazil
}

\author{
Luciane Amorim Santos, ${ }^{1}$ Joana Paixao Monteiro-Cunha, ${ }^{1 *}$ Adriano Fernando Araujo, ${ }^{1}$ \\ Carlos Brites, ${ }^{2}$ Bernardo Galvao-Castro, ${ }^{1,3}$ and Luiz Carlos Junior Alcantara ${ }^{1,3,4}$ \\ ${ }^{1}$ Advanced Public Health Laboratory, Gonçalo Moniz Research Center, Oswaldo Cruz Foundation, Salvador, \\ Bahia, Brazil \\ ${ }^{2}$ Federal University of Bahia, Salvador, Bahia, Brazil \\ ${ }^{3}$ HTLV Center/Bahia School of Medicine and Public Health / Bahia Foundation for Science Development, \\ Salvador, Bahia, Brazil \\ ${ }^{4}$ National Cancer Institute, National Institutes of Health, Bethesda, Maryland
}

The extraordinary genetic diversity and immune evasion of human immunodeficiency virus (HIV) pose significant challenges for vaccine development and antiretroviral therapy efficacy. The objective of this study was to characterize the molecular profile of HIV-1 epidemic in Salvador, Bahia, Brazil, determining the genetic subtypes and the presence of antiretroviral resistance mutations. HIV-1 pol DNA sequences from 57 individuals infected with HIV were obtained by PCR, followed by sequencing. The subtypes were determined by phylogenetic analyses and the intersubtype recombination was investigated by bootscanning. The pol subtypes were compared with gag and env subtypes. Antiretroviral susceptibility was evaluated through the Stanford HIV resistance Database. The subtypes frequencies were: $77.2 \%$ of subtype $B, 1.8 \%$ of subtype $F 1$, and $21.0 \%$ of $B F$ recombinant forms. Two intergenic and three intragenic BF recombinant patterns were observed. Six $(10.5 \%)$ viruses were related to CRF28/CRF29, two were related to CRF12 $(3.5 \%)$, and one $(1.8 \%)$ was CRF39. Fourteen $(24.6 \%)$ strains carried one or more mutations associated with at least intermediate resistance: $24.6 \%$ had resistance to nucleoside reverse transcriptase inhibitors, $21.0 \%$ to nonnucleoside reverse transcriptase inhibitors, and $7 \%$ to protease inhibitors. The substitutions I54V (7.0\%), M184V (14.0\%), and K103N (10.5\%) were the most frequent within each class of drugs. The results show a high diversity of BF genotypes and a lower prevalence of major reverse transcriptase and protease drug resistance mutations in Salvador, compared with other regions of Brazil. These findings may contribute to improve treatment strategies of patients infected with HIV-1 from this Brazilian region. J. Med. Virol. 83:2066-2072, 2011. ๑ 2011 Wiley Periodicals, Inc.

KEY WORDS: HIV-1; CRF; resistance

\section{INTRODUCTION}

Today approximately 33 million people are infected with human immunodeficiency virus (HIV) worldwide [UNAIDS, 2007]. The characteristics of the viruses found in each geographic region are different, mainly due to HIV high genetic diversity, host genetic factors, and the selective pressure of antiretroviral therapy (ARV). Because of its great variability, HIV has been classified in two types, three groups, nine subtypes [Mccutchan, 2000], 48 Circulating Recombinant Forms (CRF), and various Unique Recombinant Forms (URF) [Los Alamos HIV Database, 2011]. The distribution of these genotypes is also highly variable around the world.

After 20 years of epidemic, there is still no effective vaccine or cure for HIV and the genetic diversity of the virus is one of the major obstacles for these achievements. In despite of this, the availability of an

Grant sponsor: FAPESB; Grant number: 303/03; Grant sponsor: Brazilian Ministry of Health; Grant numbers: 306/04; 307/ 04; Grant sponsor: CNPq.

*Correspondence to: Joana Paixao Monteiro-Cunha, Laboratório Avançado de Saúde Pública, Centro de Pesquisa Gonçalo Moniz, Fundação Oswaldo Cruz.Rua Waldemar Falcão, 121, Candeal, Salvador, Bahia 40296-610, Brazil.

E-mail: jmonteiro@bahia.fiocruz.br

Accepted 1 June 2011

DOI 10.1002/jmv.22170

Published online in Wiley Online Library

(wileyonlinelibrary.com). 
increasing number of antiretroviral (ARV) agents have lead to improvement in quality of life, reduction of morbidity, and mortality with restoration and preservation of immunologic functions. On the other side, ARV therapy can also lead to drug failure [Perelson et al., 1996; Palella et al., 1998; Sepkowitz, 2001], caused mainly by mutations in the pol gene, which encodes the ARV target enzymes (protease and reverse transcriptase) [Coffin, 1995]. Therefore, the monitoring of resistance mutations, subtype prevalence, and emergence of new CRFs is important for HIV surveillance programs, controlling the transmission of strains with anti-retroviral resistance mutations, the understanding of the mechanisms by which the virus evolves and to evaluate the best treatment options for the patients.

The aims of this study were to characterize the molecular profile of HIV-1 epidemic in Salvador, Bahia, Brazil, determining the genetic subtypes and the presence of ARV resistance mutations.

\section{METHODS}

\section{Study Population}

Fifty-seven patients infected with HIV followed at the Hospital Professor Edgar Santos in Salvador, Bahia, Brazil were included in this study. Five milliliter of whole blood samples were collected during the year of 2006. Samples were stored at $-20^{\circ} \mathrm{C}$ at the Advanced Laboratory of Public Health (LASP)/ CPqGM/FIOCRUZ in Salvador, Bahia, Brazil. All patients included in this study signed the letter of informed consent. The available clinical data were collected from medical records. This study was approved by CPqGM/FIOCRUZ Ethics Committee.

\section{DNA Extraction and PCR}

The genomic DNA of all samples was extract using Qiagen extraction kit (QIAGEN, Valencia, CA). The pol fragment was amplified using a nested polymerase chain reaction (PCR). The primers used were DP10 and LR54 for the first round and DP16 and RT12 for the second round. The PCR cycling conditions were as follow: three cycles of denaturing at $95^{\circ} \mathrm{C}$ for $3 \mathrm{~min}$, annealing at $55^{\circ} \mathrm{C}$ for $1 \mathrm{~min}$ and primer extension at $72^{\circ} \mathrm{C}$ for $1 \mathrm{~min}$; another 35 cycles of denaturing at $95^{\circ} \mathrm{C}$ for $1 \mathrm{~min}$, annealing at $55^{\circ} \mathrm{C}$ for $45 \mathrm{sec}$ and primer extension at $72^{\circ} \mathrm{C}$ for $1 \mathrm{~min}$ and a final extension at $72^{\circ} \mathrm{C}$ for $10 \mathrm{~min}$. The PCR products were then purified using the Qiagen columns (QIAGEN) and sequenced in an ABI 3100 Genetic Analyzer (Applied Biosystems, Foster City, CA) using a Big Dye Terminator kit (Applied Biosystems). The primers used for sequencing were the DP16 and RT12 with the inner primers LR49 and LR51.

\section{Sequence Analyses}

The sequences were analyzed using the SeqScape v2.1.1 software (Applied Biosystems) to define the consensus sequence for each sample. We used BLAST search against the HIV-1 sequences database (http:// blast.ncbi.nlm.nih.gov) to check for contamination. The sequences were reported to GenBank under the accession numbers JN029691-JN029747.

Phylogenic analyses were performed to subtype each sequence. Reference sequences form all HIV-1 subtypes were downloaded from Los Alamos database, aligned with the new sequences using CLUSTAL-X [Thompson et al., 1997] and manually edited using GENDOC [Nicholas et al., 1997]. One sequence of the HIV-1 group O (MVP5180) was used as outgroup. Phylogenic reconstruction was performed using the PAUP $^{*}$ software v4.02 ${ }^{\mathrm{a}}$ [Swofford, 1997] to generate neighbor-joining (NJ) and maximum likelihood (ML) trees applying the GTR nucleotide substitution model with invariable sites and gamma distribution. The bootstrap value (1,000 replicates) was calculated to check the node reliability and was considered significant when above $70 \%$. To calculate de statistical support for the tree branches the likelihood-ratio test was used. All trees were visualized using FigTree v1.2.2 [available at http://tree.bio.ed.ac.uk/software]. Intersubtype recombination was identified using Bootscanning implemented in SIMPLOT software, version 2.5 [Salminen et al., 1995] and the GENEDOC software was used to analyze the crossover points by visual inspection of the alignment. Each part of the mosaic structure was confirmed by the reconstruction of bootstrapped NJ trees as described above. The $\mathrm{BF}$ recombinant forms determined were aligned with reference sequences of CRFs from the Los Alamos database and analyzed by phylogenetic reconstruction as described above. All sequences were submitted to the REGA subtyping tool [De Oliveira et al., 2005; Alcantara et al., 2009].

\section{Drug Resistance Analyses}

All sequence was submitted to the Stanford HIV resistance database [http://hivdb.stanford.edu/] to check for the presence of antiretroviral resistance mutation.

\section{RESULTS}

The genetic and epidemiological characteristics of the studied patients are described in Table I. The mean age was 42.41 ranging from 12 to 64 years. Thirty-six individuals (63.2\%) were male and 21 $(36.8 \%)$ were female. The major risk behavior identified was heterosexual transmission (59.65\%), followed by men who have sex with men (MSM) (15.79\%), vertical transmission (5.26\%), intravenous drug user (IDU) (5.26\%), bisexual (3.51\%), and blood transfusion $(1.75 \%)$. The risk behavior was unknown for $8.77 \%$ of the patients (Table I). The CD4 cell count was bellow 200 cells $/ \mu$ l in $12(21.1 \%)$ patients and the viral load was higher than 10,000 copies $/ \mathrm{ml}$ in $20(35.1 \%)$ patients. Eleven (19.3\%) patients were treatment naïve and $44(77.2 \%)$ were using ARV treatment, but the drug regimen information was only available for 
14 patients. Two patients (3.5\%) had no information regarding ARV therapy.

The protease and reverse transcriptase regions of the pol gene were used to subtype the sequences of the 57 individuals included in this study. Forty-five (78.9\%) sequences were subtype $\mathrm{B}$, three $(5.3 \%)$ were subtype $\mathrm{F} 1$ and nine (15.8\%) were $\mathrm{BF}$ recombinant (Table I). The pol subtypes were compared with the subtypes found for these samples in a previous study [Araujo et al., 2010], which used gag and env regions. Three sequences were intergenic BF recombinants. One subtype $\mathrm{B}$ sequence in pol was $\mathrm{F} 1$ in env and two subtype $\mathrm{F}$ sequences in pol were $\mathrm{BF}$ in gag and $\mathrm{B}$ in env. Therefore, the subtype prevalence in this population was $77.2 \%$ (44) of $\mathrm{B}, 1.8 \%$ (1) of F, and $21.0 \%$ (12) of $\mathrm{BF}$ recombinants.

Among the nine (15.8\%) BF recombinants detected in the pol fragment, three different mosaic patterns were observed. Six viruses (BAS008, BAS015, BAS023, BAS046, BAS069, and BAS079) showed the same recombination pattern and breakpoints in the SIMPLOT analysis and clustered together with CRF28_BF and CRF29_BF sequences. Two viruses (BAS075 and BAS081) were closely related to each other and to CRF12_BF in all analysis, presenting the same recombination profile and breakpoints and one sequence (BAS042) was classified as CRF39_BF. The sequence alignment was divided based on the related CRF breakpoints and each subregion was analyzed. NJ and ML analyses of the individual fragments confirmed the Bootscanning recombination results (Fig. 1). In order to investigate whether the BF recombinants are autochthonous or originated from other regions, we have built a NJ tree (Fig. 2) with other BF strains circulating in Bahia, as well as, with $\mathrm{BF}$ viruses from other countries and from other Brazilian regions. While this analysis contemplated sequences from different countries, such as Spain,

TABLE I. Genetic and Epidemiologic Characteristics of HIV-1-Infected Individuals From Salvador, Brazil

\begin{tabular}{lcc}
\hline & $\begin{array}{c}\text { Number of } \\
\text { Parameters }\end{array}$ & Patients $(\mathrm{n}=57)$ \\
\hline Age (years) & Percentage \\
Gender & 42.41 & \\
Male & & \\
Female & 36 & 63.2 \\
Transmission route & 21 & 36.8 \\
Heterosexual & 34 & 59.65 \\
MSM & 9 & 15.79 \\
Bisexual & 2 & 3.51 \\
Vertical & 3 & 5.26 \\
Blood transfusion & 1 & 1.75 \\
IDU & 3 & 5.26 \\
Unknown & 5 & 8.77 \\
Subtype (pot) & & 78.9 \\
B & 45 & 15.8 \\
BF & 9 & 5.3 \\
Fl & 3 & \\
\hline
\end{tabular}

IDU, intravenous drug user.

${ }^{a}$ Mean, ranging from 12 to 64 years.

${ }^{\mathrm{b}}$ Men who have sex with men.
Argentina, Uruguay, Chile, Peru, Venezuela, Paraguay and Luxembourg, most of our recombinants were closely related to other Brazilian viruses, especially to BF strains from Feira de Santana city [MonteiroCunha et al., 2011], which is located $115 \mathrm{~km}$ northwest of Salvador.

The sequence subtype was compared with the gender of the patients. The ratio of male-to-female was roughly 1:1 (five males and seven females) among individuals infected with $\mathrm{BF}$ viruses and 2:1 (30 males and 14 females) among individuals infected with subtype B viruses. The patient infected with subtype $\mathrm{F}$ was male.

Virus from $14(24.6 \%)$ patients carried one or more mutations associated with at least intermediate resistance. Among them, 12 (85.7\%) had viral load higher than 1,000 copies/ml. Dual-class drug-resistant virus was present in $3.5 \%$ of participants while triple-class drug resistant virus was present in $7.0 \%$. Forty-three (75.4\%) isolates were susceptible to nucleoside reverse transcriptase inhibitors (NRTIs), $45(79.0 \%)$ to nonnucleoside reverse transcriptase inhibitors (NNRTIs), and $53(93.0 \%)$ to protease inhibitors (PIs). The highest rates of genotypic resistance among the samples were to delavirdine (DLV) (21.1\%), nevirapine (NVP) (21.1\%), efavirenz (EFV) (21.1\%), zidovudine (AZT) $(19.3 \%)$, and lamivudine (3TC) (17.5\%). Each PI presented $7 \%$ of predicted drug resistance in this population. All patients with resistance mutations were under ARV therapy, except patient BAS028 that was not under treatment at sampling time, but had used ARVs approximately 5 years before. This patient presented the mutation V108I that causes low-level resistance to all NNRTIs except etravirine (ETR). The commonest mutation associated with PI resistance was I54V (7.0\%), while M184V (14.0\%) and K103N (10.5\%) were the commonest mutations associated with NRTI and NNRTI resistance, respectively. Minor PI mutations were also found in nine (15.8\%) sequences (Table II).

\section{DISCUSSION}

The study of the HIV-1 molecular epidemiology and diversity is of great importance for the better understanding of its epidemic in Brazil, where the subtype $\mathrm{B}$ is the most frequent, followed by $\mathrm{BF}$ recombinants and subtypes $\mathrm{F}$ and $\mathrm{C}$, with remarkable differences among the geographic regions [Morgado et al., 2002]. For instance, in the south of the country, the subtype $\mathrm{C}$ is found in a much higher prevalence than in other regions [Soares et al., 2003]. Therefore, the identification of HIV-1 circulating forms in Salvador is important for monitoring the emergence of new recombinant strains, the migration of subtype $\mathrm{C}$ from the south of the country and the introduction of new subtypes from different geographic regions. In this study, we found $77.2 \%$ of subtype B, $21.0 \%$ of BF recombinant, and $1.8 \%$ of subtype $F$. In agreement with previous studies of the Northeast Brazil epidemiology, 


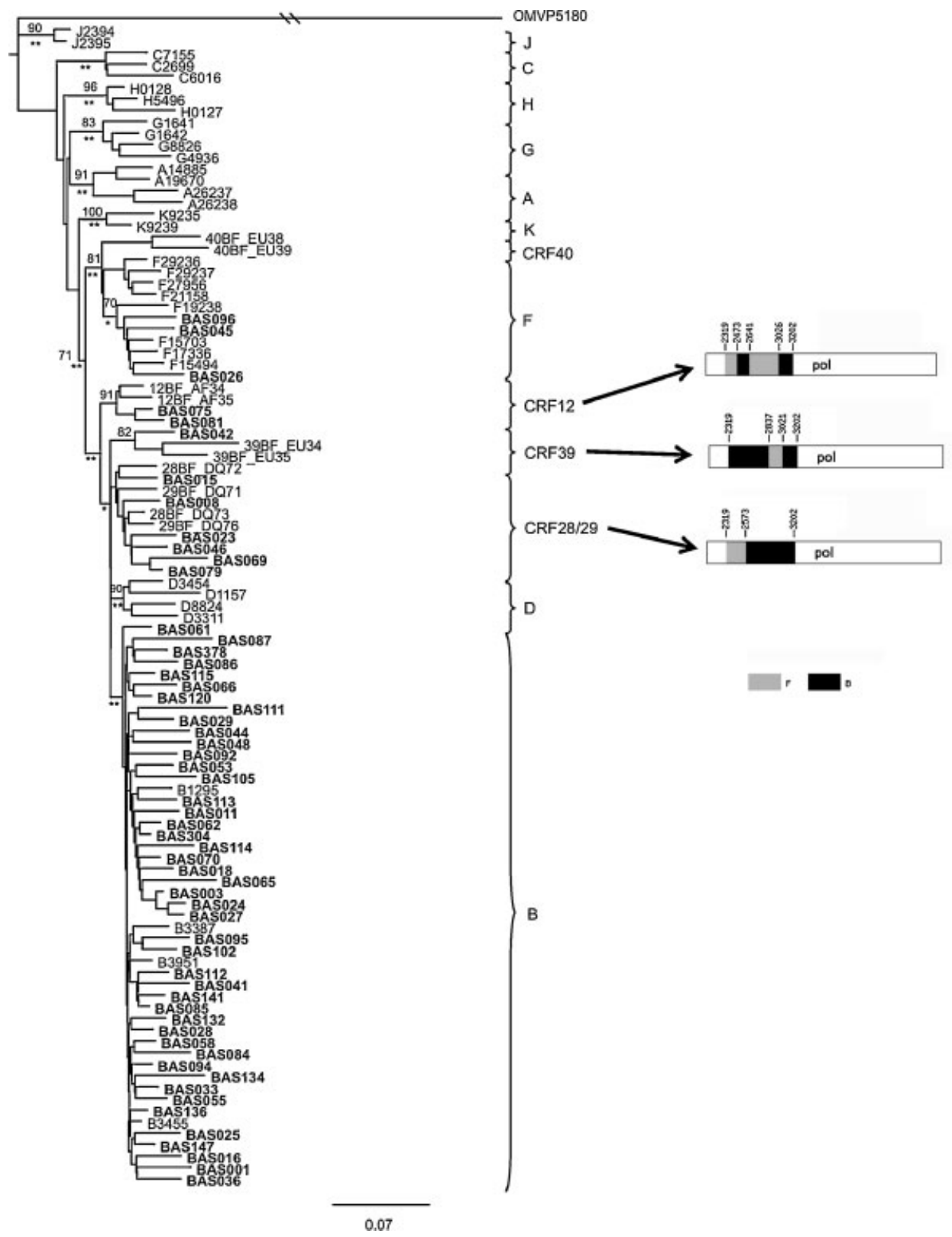

Fig. 1. Neighbor-joining (NJ) phylogenetic tree constructed using the GTR $+\mathrm{I}+\mathrm{G}$ model of nucleotides substitution, showing the phylogenic relationship among the HIV-1 strains from this study (in bold) and HIV-1 group M reference sequences from different subtypes and BF CRFs. The HIV-1 group O sequence OMVP5180 was used as outgroup. The 1,000 replicate bootstrap values that were $>70 \%$ are indicated in the branches. The ML method branches statistic support are indicated with one asterisk $\left(^{*}\right)$ when significant $(P<0.05)$, and with two asterisks $\left.{ }^{* *}\right)$ when highly significant $(P<0.001)$. The three distinct patterns of recombination between subtypes $\mathrm{B}$ and $\mathrm{F}$ are represented.

no subtype $\mathrm{C}$ was found. The subtype prevalence based on the pol, gag, and env genes was strikingly different to that described by Araujo and cols for the same population based on gag and env genes alone $(91.8 \% \mathrm{~B}, 3.3 \% \mathrm{BF}, 4.9 \% \mathrm{~F})$ [Araujo et al., 2010]. In contrast, our subtype prevalence was similar to that found in a study that also used pol, gag, and env genes $(73.9 \% \mathrm{~B}, 22.7 \% \mathrm{BF}, 2.3 \% \mathrm{~F}, 1.1 \% \mathrm{C})$ [Brennan et al., 2007]. In fact, nine intragenic recombinants identified in this study were previously classified as pure subtypes [Araujo et al., 2010]. This result reinforces the importance of multiple genomic region characterization in order to obtain a reliable profile of the HIV-1 molecular epidemiology, especially in regions with high diversity of genotypes.

Five different recombination structures were found in this population. Two of them were intergenic recombinant patterns: one represented by BAS001 $\left(\mathrm{B}_{\mathrm{pol}} / \mathrm{F} 1_{\mathrm{env}}\right)$ and the other represented by BAS026 and BAS096 $\left(\mathrm{BF}_{\mathrm{gag}} / \mathrm{F} 1_{\mathrm{pol}} / \mathrm{B}_{\mathrm{env}}\right)$. These two sequences presented the same genotype in all three genes, and therefore could be representatives of the same 


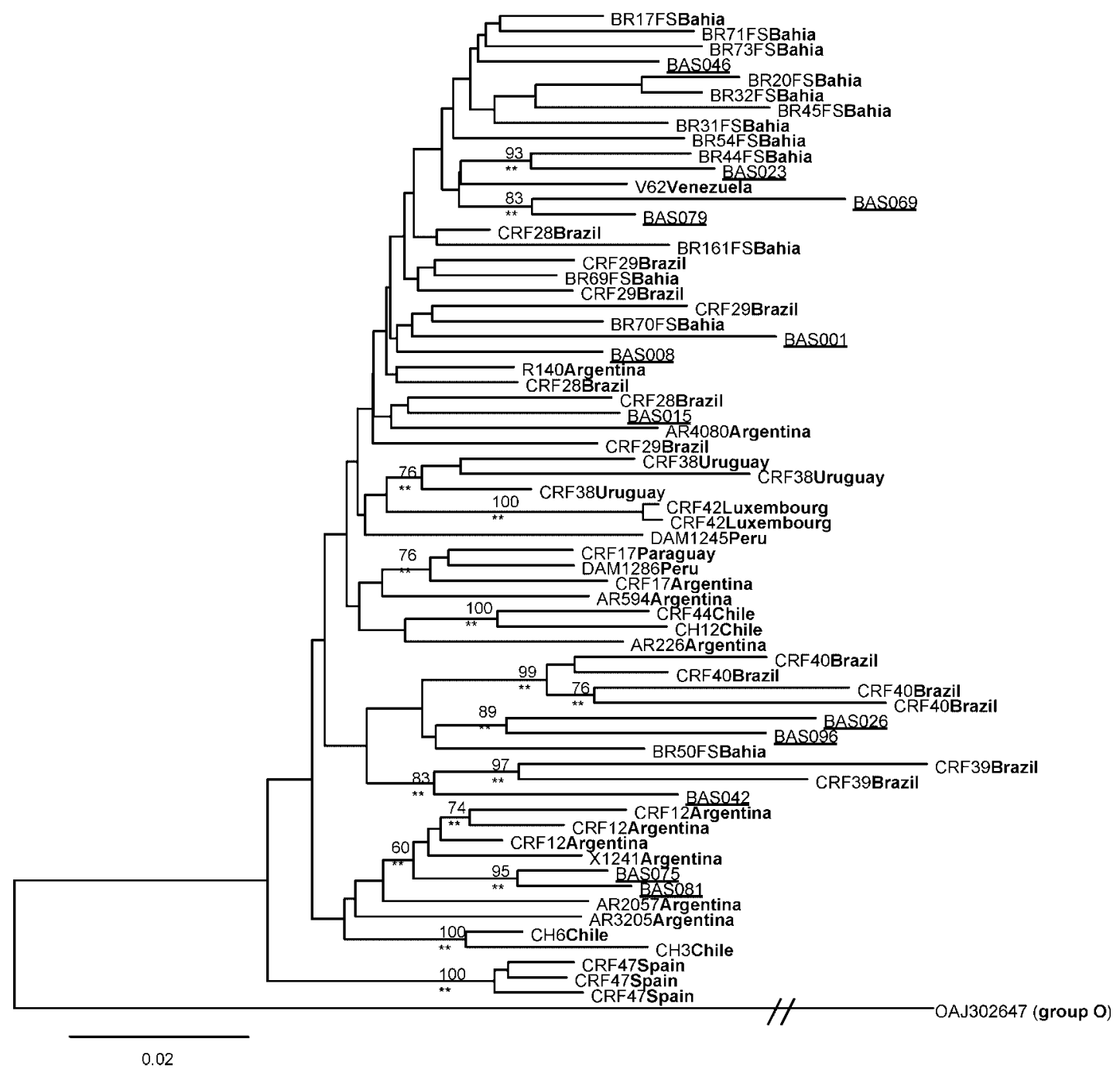

Fig. 2. pol phylogenetic relationships among HIV-1 BF recombinant viruses from different countries. A group $\mathrm{O}$ sequence was used as outgroup. The tree was constructed using the neighbor-joining method and the bootstrap values for 1,000 replicates are indicated. Branches highly supported by the ML method are indicated ( $\left.{ }^{*} P<0.001\right)$. HIV-1 sequences generated in the present study are underlined.

recombinant ancestral. However, they showed distinct intra-gag breakpoints [Araujo et al., 2010], which indicates different recombination events or, less likely, a common ancestral that went through subsequent recombination processes. In fact, BR26BA and BR96BA did not show a direct phylogenetic relationship in the trees based on gag, pol, or env fragments. The other three recombination patterns were represented by intragenic recombinant viruses. Analyzing their pol sequences, six (10.5\%) were related to CRF28/CRF29, two (3.5\%) were related to CRF12, and one (1.8\%) was related to CRF39. Out of the six CRF28/29 representatives, five were previously characterized in env (positions 6,945-8,183 relative to HXB2 genome) as subtype $B$, the same genotype found in CRF28/29 sequences [Araujo et al., 2010]. However, the gag region (positions 898-1,968 relative to HXB2 genome) of the two viruses with available sequences (BAS008 and BAS079) were also classified as pure subtype B, while CRF28/29 sequences present a crossover point (1323) in this fragment. Similarly, samples BAS075 and BAS081 showed the same recombination pattern as CRF12 in pol, but had subtype B in gag, while CRF12 have a breakpoint at position 952 and are mostly subtype $\mathrm{F}$ in this fragment. Moreover, BAS081 was B in env, while CRF12 is $\mathrm{F}$ in the same fragment. BAS075 was not amplified in gag. On the contrary, sample BAS042 showed the same recombination pattern in pol and the same env genotype (subtype F) as CRF39. When compared with BF viruses from other locations, except for samples related to CRF12 from Argentina (BAS075 and BAS081), our BF sequences formed monophyletic groups with other Brazilian sequences (Fig. 2). The above observations suggest that different BF CRFs are co-circulating in Northeast Brazil and that they are undergoing 
TABLE II. Drug Resistance-Associated Mutations in HIV-1 Strains Circulating in Salvador, Bahia, Brazil

\begin{tabular}{llllll}
\hline PI & $\mathrm{n}(\%)$ & NRTI & $\mathrm{n}(\%)$ & NNRTI & $\mathrm{n}(\%)$ \\
\hline $\begin{array}{l}\text { Mutation } \\
\text { I54V/L }\end{array}$ & $4(7.0)$ & Ml 84V & $8(14.0)$ & K103N/E/S & $6(10.5)$ \\
V82A & $3(5.3)$ & V118I & $5(8.8)$ & V90I & $3(5.3)$ \\
M46I/L & $2(3.5)$ & K70R/I & $5(8.8)$ & Y188L & $2(3.5)$ \\
L90M & $2(3.5)$ & M41L & $5(8.8)$ & V108I & $2(3.5)$ \\
L33F & $2(3.5)$ & L210W/E & $5(8.8)$ & V181C & $2(3.5)$ \\
L24I & $1(1.8)$ & D67N & $4(7.0)$ & LI 001 & $2(3.5)$ \\
F53L & $1(1.8)$ & T215Y/F & $4(7.0)$ & V106I & $2(3.5)$ \\
L23I & $1(1.8)$ & K219N/Q & $3(5.3)$ & G190A & $1(1.8)$ \\
I50V & $1(1.8)$ & T69N/A & $2(3.5)$ & V179D & $1(1.8)$ \\
N88D & $1(1.8)$ & E44D & $2(3.5)$ & & \\
A71T/V & $5(8.8)$ & D177E & $1(1.8)$ & & \\
F53V & $1(1.8)$ & T200A & $1(1.8)$ & & \\
V32M & $1(1.8)$ & R211K & $1(1.8)$ & & \\
G73C & $1(1.8)$ & K49R & $1(1.8)$ & & \\
T74S & $1(1.8)$ & I135T & $1(1.8)$ & & \\
K43T $^{\mathrm{a}}$ & $1(1.8)$ & S162C & $1(1.8)$ & & \\
N88H $^{\mathrm{a}}$ & $1(1.8)$ & A62V & $1(1.8)$ & & \\
\hline
\end{tabular}

${ }^{\mathrm{a}}$ Minor mutations.

further local recombination processes. In addition, all the intragenic recombinants identified in this study were previously classified as pure subtypes in the study based on gag and env regions [Araujo et al., 2010]. This reinforces the importance of multiple genomic region characterization in order to obtain a reliable profile of the HIV-1 molecular epidemiology, especially in regions with high diversity of genotypes.

The epidemiological data showed the heterosexual contact $(59.7 \%)$ as the most frequent transmission route, followed by men who have sex with men (15.8\%), agreeing with the Brazilian Ministry of Health Bulletin [Boletim Epidemiológico AIDS, 2010]. The general population (1.7:1) and the subtype B (2.1:1) male/female ratio were similar to the national ratio (1.6:1), while the sex ratio of the $\mathrm{BF}$ group was the opposite (0.7:1). Moreover, $91.7 \%$ of the $\mathrm{BF}$ patients were heterosexually infected. These findings suggest an association between the $\mathrm{BF}$ epidemic and heterosexual transmission $(\mathrm{OR}=11.0 ; 95 \% \mathrm{CI}$ 1.3062-92.6325) and also, between the BF epidemic and female gender $(\mathrm{OR}=3.0 ;$ 95\% CI 0.8084 11.1333 ), as previously reported [Rios et al., 2005; Monteiro et al., 2009].

In this study, $24.6 \%$ of the strains showed some level of resistance to NRTIs, $21.0 \%$ to NNRTIs and $7.0 \%$ to PIs. The substitutions I54V $(7.0 \%)$, M184V (14.0\%), and $\mathrm{K} 103 \mathrm{~N}$ (10.5\%) were the most frequent within each class of drugs. These mutations were previously related in a significant higher prevalence among patients under multiantiretroviral therapy or with treatment failure in other regions of Brazil [Couto-Fernandez et al., 2005; de Sa-Filho et al., 2008; Toledo et al., 2010], with I54V varying from $20 \%$ to $24 \%$, M $184 \mathrm{~V}$ from $68 \%$ to $88 \%$ and K103N from $28 \%$ to $52 \%$. This could be related with the fact that the rising of resistance mutations follows the sequential use of ARVs, which started earlier and in a more comprehensive way in the Southeast Region of Brazil. In this study, the mean time of ARV exposure of the patients presenting drug resistance mutations was 7 years.

The results show a high diversity of BF genotypes, particularly the co-circulation of three distinct CRFs and a lower prevalence of major reverse transcriptase and protease drug resistance mutations in Salvador, compared with other regions of Brazil. These findings contribute for better understanding the HIV-1 molecular epidemiology and may also contribute to improve treatment strategies of patients infected with HIV-1 from this Brazilian region.

\section{ACKNOWLEDGMENTS}

Bioinformatics analysis was performed at the LASP/CPqGM/FIOCRUZ Bioinformatics Unit. JPMC thank FAPESB for a postdoctoral fellowship.

\section{REFERENCES}

Alcantara LC, Cassol S, Libin P, Deforche K, Pybus OG, Van Ranst M Galvão-Castro B, Vandamme AM, de Oliveira T. 2009. A standardized framework for accurate, high-throughput genotyping of recombinant and non-recombinant viral sequences. Nucleic Acids Res 37: (Web Server issue): W634-W642.

Araujo FA, Brites C, Monteiro-Cunha JP, Santos LA, Galvão-Castro B, Alcantara LCJ. 2010. Lower prevalence of human immunodeficiency virus Type 1 Brazilian subtype B found in Northeastern Brazil with slower progression to AIDS. AIDS Res Hum Retroviruses 26:1249-1254.

Boletim Epidemiológico AIDS. 2010. Available at http://www.aids gov.br/pagina/boletim-epidemiologico. Accessed on February 2011.

Brennan CA, Brites C, Bodelle P, Golden A, Hackett J, Jr, Holzmayer V, Swanson P, Vallari A, Yamaguchi J, Devare S, Pedroso C, Ramos A, Badaro R. 2007. HIV-1 strains identified in Brazilian blood donors: Significant prevalence of B/F1 recombinantes. AIDS Res Hum Retroviruses 23:1434-1441.

Coffin JM. 1995. HIV population dynamics in vivo: Implictions for genetics variation, pathogenesis, and therapy. Science 267:483489.

Couto-Fernandez JC, Silva-de-Jesus C, Veloso VG, Rachid M, Gracie RS, Chequer-Fernandez SL, Oliveira SM, Arakaki-Sanchez D, Chequer PJ, Morgado MG. 2005. Human immunodeficiency virus type 1 (HIV-1) genotyping in Rio de Janeiro, Brazil: Assessing subtype and drug-resistance associated mutations in HIV-1 infected individuals failing highly active antiretroviral therapy. Mem Inst Oswaldo Cruz 100:73-78.

De Oliveira T, Deforche K, Cassol S, Salminen M, Paraskevis D, Seebregts C, Snoeck J, van Rensburg EJ, Wensing AM, van de Vijver DA, Boucher CA, Camacho R, Vandamme AM. 2005. An automated genotyping system for analysis of HIV-1 and other microbial sequences. Bioinformatics 21:3797-3800.

de Sa-Filho DJ, Soares Mda S, Candido V, Gagliani LH, Cavaliere E, Diaz RS, Caseiro MM. 2008. HIV type 1 pol gene diversity and antiretroviral drug resistance mutations in Santos, Brazil. AIDS Res Hum Retroviruses 24:347-353.

Los Alamos HIV Database. HIV Circulating Recombinant Forms (CRFs). Available at http://www.hiv.lanl.gov/content/sequence/ HIV/CRFs/CRFs.html. Accessed on February 2011.

Mccutchan FE. 2000. Understanding the genetic diversity of HIV-1. AIDS 14:S31-S44.

Monteiro JP, Alcantara LCJ, De Oliveira T, Oliveira AM, Melo MAG, Brites C, Galvao-Castro B. 2009. Genetic variability of human immunodeficiency virus-1 In Bahia State, Northeast, Brazil: High diversity of HIV genotypes. J Med Virol 81:391-399.

Monteiro-Cunha JP, Araujo AF, Santos E, Galvao-Castro B, Alcantara LC. 2011. Lack of high-level resistance mutations in HIV Type $1 \mathrm{BF}$ recombinant strains circulating in Northeast Brazil. AIDS Res Hum Retroviruses 27:623-631. 
Morgado MG, Guimarães ML, Galvão-Castro B. 2002. HIV-1 polymorphism: A challenge for Vaccine Develoment. Mem Inst Oswaldo Cruz 97:143-150.

Nicholas KB, Nicholas HBJ, Deerfield DW. 1997. GeneDoc: Analysis and visualization of genetic variation. EMB News 14:30.

Palella FJ, Jr, Delaney KM, Moorman AC, Loveless MO, Fuhrer J, Satten GA, Aschman DJ, Holmberg SD. 1998. Declining morbidity and mortality among patients with advanced human immunodeficiency virus infection. HIV Outpatient Study Investigators. N Engl J Med 338:853-860.

Perelson AS, Neumann AU, Markowitz M, Leonard JM, Ho DD 1996. HIV-1 dynamics in vivo: Virion clearance rate, infected cell life-span, and viral generation time. Science 271:1582-1586.

Rios M, Fernandez J, Jaramillo P, Paredes V, Sanchez JL, LagunaTorres VA, Carr JK, Ramirez E. 2005. Molecular epidemiology of HIV type 1 in Chile: Differential geographic and transmission route distribution of $\mathrm{B}$ and $\mathrm{F}$ subtypes. AIDS Res Hum Retroviruses 21:835-840.

Salminen MO, Carr JK, Burke DS, Mccutchan FE. 1995. Identification of breakpoints in intergenotypic recombinants of HIV type 1 by bootscanning. AIDS Res Hum Retroviruses 11:1423-1425.
Sepkowitz KA. 2001. AIDS-The first 20 years. N Engl J Med 344: 1764-1772.

Soares MA, De Oliveira T, Brindeiro RM, Diaz RS, Sabino EC, Brigido L, Pires IL, Morgado MG, Dantas MC, Barreira D, Teixeira PR, Cassol S, Tanuri A. Brazilian Network for Drug Resistance Surveillance. 2003. A specific subtype C of human immunodeficiency virus type 1 circulates in Brazil. AIDS 17:1121.

Swofford D. 1997. PAUP*: Phylogenetic analysis using parsimony. Version 4.0b10. Smithsonian Institution. Washington, D.C.

Thompson JD, Gibson TJ, Plewniak F, Jeanmougin F, Higgins DG. 1997. The ClustalX windows interface: Flexible strategies for multiple sequence alignment aided by quality analysis tools. Nucleic Acids Res 25:4876-4882.

Toledo PV, Carvalho DS, Romagnoli L, Marcinko G, Cunha CA, Souza MN, Brindeiro R, Queiroz-Telles F. 2010. HIV-1 genotypic resistance profile of patients failing antiretroviral therapy in Paraná, Brazil. Braz J Infect Dis 14:360-371.

UNAIDS. AIDS epidemic update 2007. Available at http://www.unaids. org/en/KnowledgeCentre/HIVData/EpiUpdate/EpiUpdArchive/ 2007/default.asp. Accessed on February 2011. 